$\xi=-$ 国

\title{
An IoT Framework for Real-Time Event Detection and Acquisition Using Mobile Sink
}

\author{
Samaleswari Prasad Nayak ${ }^{1}$. Satyananda Champati Rai ${ }^{2}$. Sateesh Kumar Pradhan ${ }^{3}$ \\ ${ }^{I}$ Dept. of Computer Science and Engineering, Silicon Institute of Technology, Bhubaneswar, Odisha, India \\ ${ }^{2}$ Dept. of Information Technology, Silicon Institute of Technology, Bhubaneswar, Odisha, India \\ ${ }^{3}$ PG Dept. of Comp. Sc. \& Application, Utkal University, Bhubaneswar, Odisha, India \\ * Corresponding author E-mail: samaleswari.nayak@silicon.ac.in
}

\begin{abstract}
Immediate service provisioning with real-time attention to a critical patient at remote locations is a challenging task for health care unit personnel. Several incidents remain unattended for long duration due to tracking difficulties to the exact place of event. During accidents, natural calamities, any kind of disaster, providing the basic services to the affected people is a great challenge to the persons present nearby the location. This requires a real-time event status report collection, monitoring condition, arrange and provision system in place. Similarly during critical situation of a patient in hospital if the user requires blood platelet and plasma from a different centre to current place, which may not possible due to heavy traffic in the locality. In this regard we propose a quadcopter based mobile sink to navigate to the place, track, monitor and inform to the remote server which can also be treated as an intra-city delivery model. A gamepad is used to control the quadcopter from the nearby place, its movement mechanism is controlled by the integration of different sensors and other components. Several applications have been used in our model to control the position of quadcopter, capture images through sensors and forward the information to the destination to achieve the objective of real time data acquisition and assistance at the unreachable place of the event, with minimal delay.
\end{abstract}

Keywords: Mobile Sink, Monitoring, Quadcopter, Tracking, WSN

\section{Introduction}

As stated by authors in[1,2] a wireless sensor network (WSN) is a collection of sensors, which have limited battery power and are capable of performing communication among themselves within certain ranges .The deployed sensors are expected to sense different types of events and to collect the sensed data from the network. The received data are required to be further transmitted to the neighbouring nodes. According to the idea used by Bachrach et al. [3], data transmission consumes highest amount of energy compared with other tasks such as receiving, processing and sensing in a wireless environment. Two fields are reserved in a data packet to store the position and remaining battery power of the sensor. As stated by Carrillo et al. [4], sink can able to determine the position of all nodes after receiving data packets from them for relative processing. The sensor having maximum residual energy is being selected by the sink [5] and for optimized energy consumption as well as load balancing in the network is suggested by Seung H. et al. [6] which is essential for extending the network lifetime.

Our proposed model has multiple clusters and mobile sink for real time event detection and tracking. It can be deployed at road side to detect, monitor incidents and to assist the affected persons. Authors through [7,8] provided the idea for providing the sink mobility concept to deliver the data from one place to another. Intelligent Traffic Light system (ITLS) [5, 7] mechanism has been proposed for auto detection of accident and rescuing the affected by ambulance in real time. In addition Chen $\mathrm{N}$, et al. [8] suggested inclusion of a control unit to determine the path and display the route to the ambulance to reach the hospital safely. The controller is smart enough to detect the place of the accident and an ambulance will reach by the controller to assist the people suffered in the accident. To reach the hospital the automated system provides the solution. However in the existing mechanism proposed through article on real time data capturing and acquisition $[9,10]$, the measurement of the gravity to the incident place is not specified, where as analysis is done on flying of the object from one location to another. In our proposed model we have described a sink mobility approach for intra-city communication as well as to reach unreachable places for data acquisition and data aggregation using an IoT based framework.

\section{Related Work}

A mobile sink collects all geographical information from different sensors to improve the network lifetime by changing its position due to hovering. Zhao et al. has provided a flight control solution which can aggregate the collected data for further processing but theoretical analysis sometimes shows that the combining of MPC and robust feedback linearization principle does not provide any benefit for better network lifetimes as mechanism has been used to find the way for hovering without weight management [10]. To improve the network life time the author has used mobile sink in their model which achieve this through load balancing of the network [11]. The authors also have proposed a mobility model MPC to utilize effective data acquisition mechanism through sink mobility. 
In the clustering model [10] limited battery powered sensors were used where it has been restricted to improve the network lifetime due to higher amount of energy consumption during data transfer by mobile sink. The framework suggested in $[12,13]$ the node with highest load dies early and the entire communication stops in the network. To deliver data from source to destination in real time, a quadcopter with different functionalities have been designed in our work.

To address the problem of data acquisition and real time assistance to the needful, different mechanisms have been developed using drones in various fields. Using wireless audio and video system the authors have designed the virtual model which provides a solution for movement of the quadcopter in a shorter range in flying mode $[14,15]$. Here it has been discussed to stabilize the movement of the quadcopter in uneven atmospheric flow of air using PID control. In this proposed model six degrees of freedom have been implemented by considering three translational and others as rotational. Also to get better result in terms of hovering in the sky addition to few independent parameters along with coupling of the translational and rotational motions are being done. Like ground vehicles it has been used like [3, 10] how quadcopter generates little friction to prevent the motion and unable to move a longer distance according to the second law of Newton. So a novel flight control mechanism has been integrated in our model for long distance delivery of required materials to the destination.

Papa et al. [16] have made a suggestion to follow two different approaches for deployment of mobility feature with sink using unmanned aerial vehicle (UAV) mechanism. Huston et al. have given an attempt [17] that instead of having knowledge of the entire network, the sink can use circuit switching approach and forward the data packets to the destination. Otherwise by using the packet switching mechanism the sink has to collect total information from source to destination and then the movement decision can be taken by collecting information from the required sensors. The node with the maximum energy is permitted to show the route from its point to the next node and finally to the destination. The movement of the quadcopter depends on the predefined route from source to destination. Also it has to use some decision making mechanism for safe delivery by quadcopter in various applications. So the sensors with limited battery power are bottlenecked to prolong the life of the network due to the data acquisition and processing. Thus majority of current research work is targeted towards finding different solutions to provide a better idea for maximization of network life [18, 19]. As more energy being consumed during communication process the author in this article has followed multi-hop clustering approach and by using the concept of sleep based scheduling the sink can collect data from each node which results in network life enhancement. Many ideas have been proposed with a principle of quadcoptor based mobile sink [20] to collect sensor data from the WSN and deliver the object by capturing the route to provide real time assistance for various situations. By following the properties of the mobile sink node, the authors have investigated theoretically the relevant problems of path, speed, altitude and data amount what the sink node can forward [21]. By using statistics in our article we have designed a data acquisition principle based on the flight control along with carrying object, speed and altitude of the controlled mobile node. The result provides the relativity between flying time, transmission delay, packet loss rate and the mobile trajectory, velocity and relative route over the ideas $[22,21]$ which are inputs for development of the designed applications.

\section{Proposed Model}

For achieving real time data aggregation different constraints as movement trajectory, speed, altitude of the controllable mobile sink node are to be considered during designing of framework. Formulation of model is used for moving speed, height, the amount of data and delay, provides a technique for such network environment which also analyzes the performance of the mobile sink node for data fusion strategy $[23,11]$. So during critical tie applications a mobile sink model can be considered as an ideal solution for intra-city or intercity delivery of certain life saving components. The intra city communication can be possible through proposed model as the route can be managed by hovering the quadcopter, by processing the image and getting the real time locations through GPS module [24]. The quadcopter consists of four arms and four motors for hovering. To vary the speed motors are connected to the ESC. These ESC's are connected to controller board which is connected to the arduino [13, 17, 25]. The arduino receives signal from Radio Controller Transmitter and also from four ultrasonic sensors for detecting any things in different directions. The quadcopter takes an alternate path and flies by changing the motor speed when an obstacle is detected in the medium.

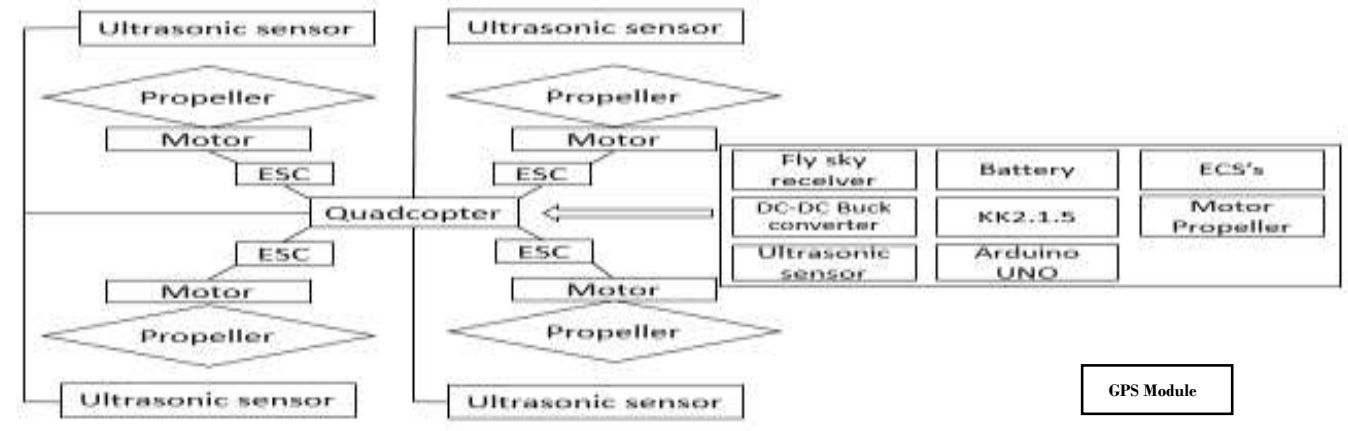

Figure-1: Proposed Model block diagram

The purpose of this section is to calculate the relationships between the thrust forces created by each propeller and the quadcopter's different movements [7, 19]. The spatial orientation can be described as the combination of three angles: roll, pitch and yaw. The roll angle $(\varphi)$ represents the rotation around the front-toback axis, the pitch angle $(\theta)$ represents the rotation around the side-to-side axis and the yaw angle $(\psi)$ represents the rotation around the vertical axis. To measure these angles it is necessary to define the axes of the reference system, according to one of the two possible approaches.
All quadcopters are made of similar shape and has four motors, and has two configurations: " $X$ " and "+" quadcopters. The difference between the two groups is the direction of displacement relative to the frame geometry. In our model we have used the " $\mathrm{X}$ " quadcopter model which considers the distances between each motor and the axes $\mathrm{X}$ and $\mathrm{Y}$ represents the distances of the arm multiplied by two different factors. As the angle between each arm is 900 , these factors are equal to $\cos 45^{\circ}$ and $\sin 45^{\circ}$. By applying the angular momentum conservation law equations, the expressions for the angles result in: Roll: 


$$
\left(\frac{d w}{d t}\right)_{A x i s x}=\frac{\left(L^{C F}+B^{C F}-F^{C F}-R^{C F}\right) \cdot D \cdot \cos 45}{I_{x x}}
$$

Pitch:

$\left(\frac{d w}{d t}\right)_{\text {Axis } Y}=\frac{\left(L^{C F}+B^{C F}-F^{C F}-R^{C F}\right) \cdot D \cdot \sin 45}{I_{y y}}$

Yaw:

$\left(\frac{d w}{d t}\right)_{\text {Axis Z }}=\frac{M_{1}+M_{3}-M_{2}-M_{4}}{I_{z z}}$

Where

$\mathrm{W}=$ Angular speed

$\mathrm{F}^{\mathrm{Cf}}, \mathrm{B}^{\mathrm{Cf}}, \mathrm{R}^{\mathrm{Cf}}, \mathrm{L}^{\mathrm{Cf}}$ : are body control force generated by front, rear, right and left motor

$\mathrm{M}_{1}, \mathrm{M}_{2}, \mathrm{M}_{3}, \mathrm{M}_{4}$ : Thrust generated by front, right, rear and left motor

$\mathrm{I}_{\mathrm{x} x}, \mathrm{I}_{\mathrm{yy}}, \mathrm{I}_{\mathrm{zz}}=$ Moment of inertia w.r.to front to back, side to side and vertical axis

\section{Components of the Proposed Model}

The major components of the mobile sink are the different sensors such as accelerometer, barometer and gyroscope. The accelerometer is being used for measurement of linear acceleration where as barometer is designed to know the height of quadcopter from ground by measuring the pressure of air and as it changes with altitude. The gyroscope is used to measure angular acceleration on an $\mathrm{x}, \mathrm{y}$, or $\mathrm{z}$ axis. Basically, it is responsible for allowing the quadcopter to fly in a stable manner. GPS is used for real-time location monitoring, management of mobile sink as well as navigation and positioning of target object remotely.

To make the model cost effective instead of using several sensors only three are selected to fulfill the requirement. In our model we have proposed to use a smart phone having these three sensors. This can be user friendly as most of the users have such a device and can be used at any time. Then the main problem arises how to read the values of these three sensors and how to connect a smart phone to a quadcopter. To overcome these problems we have used a special type of arduino chip known as arduino Mega ADK which is easily connected to both smart phone and a quadcopter. But for calculation of maximum flight time several factors like mass, power, pressure, speed, image capturing and processing are required.

For modeling, different parts of the devices are being connected with specified pins of the arduino board. As per Figure-2 the pin configuration specifies the connection of different sensors, battery, ESC and others with respective input output devices.

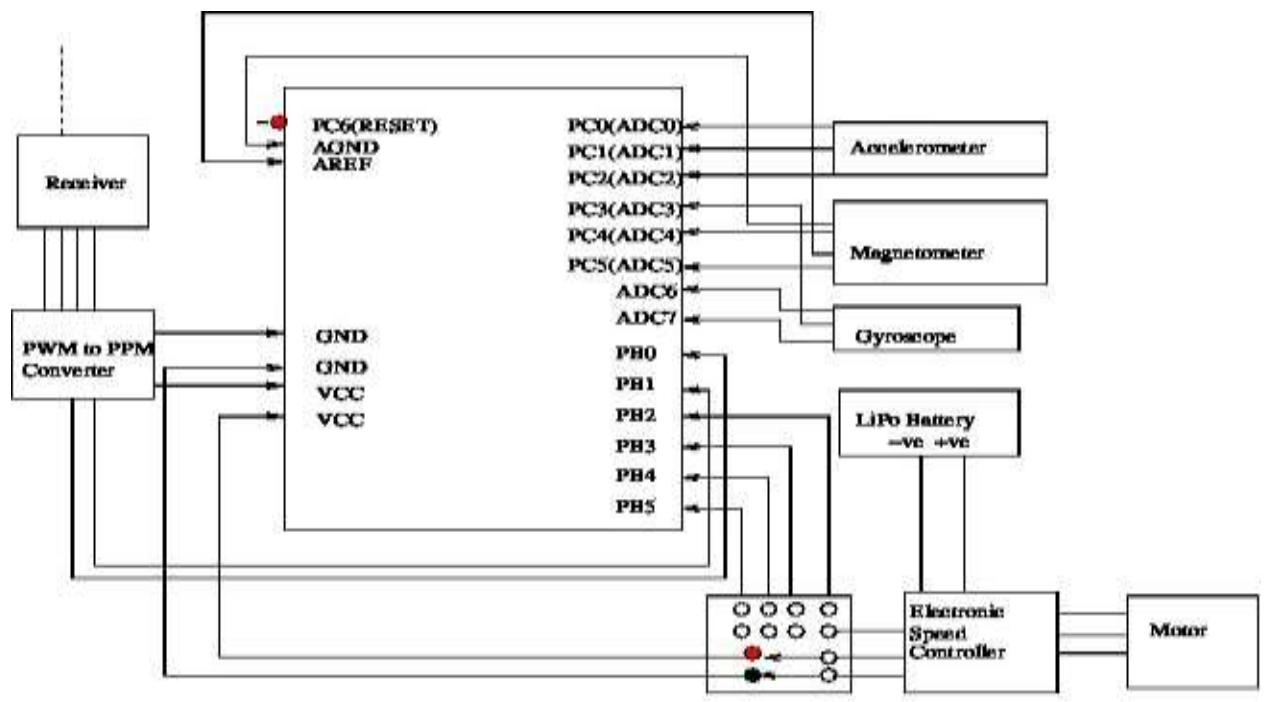

Figure 2: Pin Configuration of Model Architecture

\subsection{Force that Each Propeller Must Generate in a Hovering Situation}

We have considered a simple frame where dimensions are defined by the length, width and height of the frame. This structure must hold the effects of the thrust forces and momentums created by each propeller and the total weight of the system. It has been assumed that motors 1 and 3 spin counter-clock-wise, therefore their momentums have opposite sense to motors 2 and 4 .

According to Newton's second law, the acceleration of quadcopter towards the vertical direction is represented as:

$$
\begin{aligned}
\text { acceleration }=\frac{\sum \text { Force of motors }}{\text { mass }_{\mathrm{qd}}} & \\
& =\frac{\mathrm{F}^{\mathrm{CF}}+\mathrm{R}^{\mathrm{CF}}+\mathrm{B}^{\mathrm{CF}}+\mathrm{L}^{\mathrm{CF}}-\mathrm{w}}{\operatorname{mass}_{\mathrm{qd}}}
\end{aligned}
$$

Where $\mathrm{w}$ is treated as angular speed

In a hovering situation the quadcopter acceleration is equal to zero and the thrust created by the propellers must compensate the gravitational force. Assuming that each rotor creates the same vertical thrust, this force can be described in terms of mass:

$$
\text { Force }=\frac{W}{4}=\frac{\text { mass }_{\mathrm{qd}}}{4} * \text { ga }
$$

Where $\mathrm{ga}=$ acceleration due to gravity

The total mass of a quadcopter is the sum total masses of all the integrated components. Themodel includes 4 frames, the 4 motors and propellers, the LiPo battery, the electronic installation and the object it carries.

$$
\begin{aligned}
\operatorname{mass}_{\mathrm{qd}}=\operatorname{mass}_{\mathrm{FM}} & +4 * \operatorname{mass}_{\mathrm{MTR}}+\operatorname{mass}_{\mathrm{BATTERY}} \\
& +\operatorname{mass}_{\mathrm{ELEC}}+\operatorname{mass}_{\mathrm{PLT}} \\
& +\operatorname{mass}_{\mathrm{PLSM}}
\end{aligned}
$$

Where

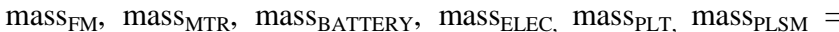
Mass of the frame, each motor, battery, all electric equipments, blood platelet and blood plasma 


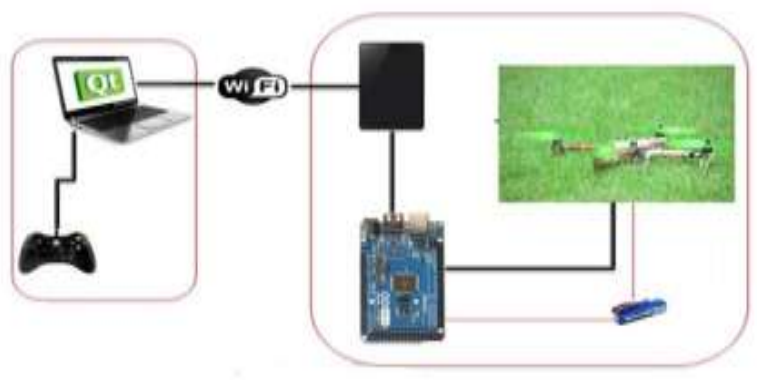

Figure3: Proposed Experimental Model

\subsection{Frame Mass Calculation}

Assuming the volume of the simplified shape, its mass can be calculated as the sum of the masses of the four arms. Mass of a single arm can be defined as

$$
\begin{aligned}
\operatorname{mass}_{\mathrm{ARM}} & =\rho_{\mathrm{FM}} \cdot \mathrm{V}_{\mathrm{ARM}} \\
& =\rho_{\mathrm{FM}} \cdot(\text { length } \cdot \text { width } \cdot \text { height }) \\
\text { mass }_{\mathrm{FM}} & =4 \text {. } \text { mass }_{\mathrm{ARM}} \\
& =4 \cdot \rho_{\mathrm{FM}} \cdot(\text { length } \text {. height } . \text { width) }
\end{aligned}
$$

Where, $\mathrm{V}_{\mathrm{ARM}}=$ Volume of each arm, $\rho_{\mathrm{FM}}=$ Density of frame In order to relate the parameters "height" and "width" with the distance "length", it is necessary to set a maximum vertical displacement that can be considered acceptable. As each arm is treated as a fixed beam, Zhang et al. [5] has suggested that this displacement can be calculated through the Euler-Bernoulli equation

disp $=\frac{\text { length }^{3} . \text { Force }}{3 \cdot \text { I. } . Y}$

\subsection{Mass of Motors and Propellers}

The mass of propellers may vary for different types, as the mass depends on its material and on its dimensions. There is no proportional relationship applicable to every propeller for real time implementation. So the mass of propeller is added with the mass of motor in all cases.

\subsection{Mass of Lipo Battery}

The capacity of a battery is directly proportional to its physical size and weights [7]. Considering this idea the mass of a LiPo battery can be increased linearly with capacity given by the subsequent equation:

$$
\mathrm{m}_{\mathrm{BAT}}=\mathrm{A} \cdot \text {. Capacity }+\mathrm{B}
$$

The parameters A and B depend on the number of cells, and their values can be seen from the manual of batteries.

\subsection{Electronic Installation Mass}

Our model consists of a controller, ESCs and the wires. The ESC modules are of light weight compared with the system mass. Depending on the different manufacturers, its mass varies between 10 to 20 grams. As the effect of these devices is not very significant in the system total mass calculation, they are considered to be 15 grams for every quadcopter and arduino UNO is considered with weight 30 grams. The wires mass calculations depend on the frame size, as the wires cover the distance between each ESC and the battery. In order to improve the quadcopter stability, the battery is always being placed at the centre of the frame. As the wires are made of cooper, who has a linear density of $7.037 \mathrm{~g} / \mathrm{m}$ and that there are two wires per arm, the total mass of 4 arms of the designed model is: $\begin{array}{rl}\text { mass }_{\mathrm{WIRES}}=4 * 2 & 2 \text { length } * 7.037 \\ & =\text { length } * 56.29\end{array}$

Where "length" is expressed in meters

The complete expression for the electronic installation mass results in:

$\operatorname{mass}_{\mathrm{ELEC}}=30+4 * 15+$ length $* 56.29=90+56.29 *$

length

The qudcoptor along with all accessories can move from one location to another location with the total mass as given in equation 12

\subsection{Power Consumed and Torque Created by the Motors in a Hovering Situation}

The brushless motors are used for our proposed quadcopter model, where the torque produced for electronic motors is expressed as

$\mathrm{T}=\mathrm{Kt}(\mathrm{I}-\mathrm{Io})$

Where $\mathrm{T}$ : Motor torque, I : Input Current, $\mathrm{I}_{0}$ : Current when there is no load on the motor, $\mathrm{K}_{\mathrm{t}}$ : Torque proportionality constant

The voltage used for motor is the sum of the back-EMF and some resistive loss.

$\mathrm{V}=\mathrm{IRm}+\mathrm{K}_{\mathrm{v}} \mathrm{W}$

Where $\mathrm{V}$ : the voltage drop across the motor, $\mathrm{R}_{\mathrm{m}}$ : is the motor resistance, $\mathrm{w}$ : the angular velocity of the motor,

$\mathrm{Kv}$ : proportionality constant.

By using this description, the power consumed by motor is calculated as

Power $=\mathbf{P}=\mathbf{I V}$
$=\frac{\left(\mathbf{T}+\mathbf{K}_{\mathbf{t}} \mathbf{I}_{\mathbf{0}}\right)\left(\mathbf{K}_{\mathbf{t}} \mathbf{I}_{\mathbf{0}} \mathbf{R}_{\mathbf{m}}+\mathbf{T} \mathbf{R}_{\mathbf{m}}+\mathbf{K}_{\mathbf{t}} \mathbf{K}_{\mathbf{v}} \boldsymbol{\omega}\right)}{\mathbf{K}_{\mathbf{t}}^{2}}$

For the purposes of our simple model, we have assumed a negligible motor resistance. Then, power is derived to be proportional to the angular velocity:

$$
\mathrm{P} \approx \frac{\left(\mathrm{T}+\mathrm{K}_{\mathrm{t}} \mathrm{I}_{0}\right) \mathrm{K}_{\mathrm{v}} \omega}{\mathrm{K}_{\mathrm{t}}}
$$

Further simplifying our model, we assume that $\mathrm{KtI}_{0}<<\mathrm{t}$ during no load on the device. As $I_{0}$ is the current when there is no load which in turn generates negligible torque can be considered generating constant power. In practice, this approximation holds well enough to find the simpler derivation for power.

$\mathrm{P} \approx \frac{\mathrm{K}_{\mathrm{v}}}{\mathrm{K}_{\mathrm{t}}} \tau \omega$

The power is used to keep the quadcopter in the air. By conservation of energy, we know that the energy the motor expends in a given time period is equal to the force generated on the propeller. So the distance in the air it displaces is $(\mathrm{P} * \mathrm{dt}=\mathrm{F} *$ $\mathrm{dx}$ ). Equivalently, the power is equal to the thrust times the air velocity

$$
P=F\left(\frac{d x}{d t}\right)
$$

As the vehicle speed is low the equation for hovering velocity as a function of thrust can be defined by representing through the term $\mathrm{v}_{\mathrm{h}}$ as 
$=\sqrt{\frac{T}{2 \rho A}}$

Where $\rho$ is the density of the surrounding air, A is the area swept out by the rotor

Using equation 17 for derivation of power, we can write

$\mathrm{P}=\frac{\mathrm{K}_{\mathrm{v}}}{\mathrm{K}_{\mathrm{t}}} \mathrm{T} \omega=\frac{\mathrm{K}_{\mathrm{v}} \mathrm{v}_{\mathrm{h}}}{\mathrm{K}_{\mathrm{t}}} \mathrm{T} \omega$

$=P=\frac{\mathrm{T}^{\frac{3}{2}}}{\sqrt{2 \rho \mathrm{A}}}$

For the thrust magnitude $\mathrm{T}$, the thrust is being proportional to the square of angular velocity of the motor and by using the previous values of the equation 20 for total torque along with a constant $b$ is derived as

$\mathrm{T}=\left(\frac{\mathrm{K}_{\mathrm{v}} \mathrm{v}_{\mathrm{h}} \sqrt{2 \rho \mathrm{A}}}{\mathrm{K}_{\mathrm{t}}} \omega\right)^{2}=\mathrm{b} \omega^{2}$

The computation value of frictional force on the quadcopter is expressed after derivation of torque. Each rotor contributes some torque about the body w.r.to any axis. This torque is required to keep the propeller spinning and providing thrust and creates the instantaneous angular acceleration and overcomes the frictional drag forces. The drag equation from fluid dynamics used for frictional force to move the model as

$F_{D}=\frac{1}{2} \rho C_{D} A u^{2}$

Where $\rho$ : the surrounding fluid density, A: is the reference area CD: a drag co-efficient

This is only accurate in some cases and provides good result for our model. The torque generated due to drag is derived as:

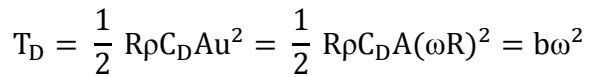

Where $\omega$ : the angular velocity of the propeller, R: the radius of the propeller, $\mathrm{b}$ : some appropriately dimensioned constant

The total torque about the $\mathrm{z}$ axis is given by the sum of all the torques from each propeller:

$\mathrm{T}=\mathrm{b}\left(\mathrm{w}_{1}^{2}-\mathrm{w}_{2}^{2}+\mathrm{w}_{3}^{2}-\mathrm{w}_{4}^{2}\right)+\mathrm{I}_{\mathrm{M}} \mathrm{W}$

Where $\mathrm{I}_{\mathrm{M}}$ : Moment of inertia of the motor about an axis

\subsection{Calculation of Pressure}

For calculating pressure of the quadcopter both static as well as dynamic pressure has been considered which again depends on altitude and air velocity. Applying the Bernoulli's equation, the expression for the total air pressure depending on the velocity is carried out in the form of

$P=p+\frac{1}{2} \rho_{A I R} v^{2}$

Where $\mathrm{P}$ is the total pressure, $\mathrm{p}$ : the static pressure, $\mathrm{v}$ : the air speed, $\rho_{\text {AIR }}$ is the air density

As we have considered as altitude is same for air before the propeller and after the propeller to maintain same static pressure at both points so considering this negligible difference the value of pressure is derived during hovering and the speed of the air before entering into the propeller is zero. Also the generated total pressure with exit velocity is :
$P=p+\frac{1}{2} \rho_{A I R} \cdot v_{E}^{2}$

Where $\mathrm{v}_{\mathrm{E}}$ : exit velocity.

\subsection{Maximum Vertical and Horizontal Speeds}

Assuming that the model will move only on the vertical direction, the expression that defines this situation is:

$$
T-F_{D R A G}=T-m \cdot g-\frac{1}{2} \rho_{A I R} \cdot C_{D} \cdot A \cdot v^{2}=m \cdot a
$$

The maximum thrust force is calculated as the combination of the maximum force that the propellers can created due to different components and mass of devices is:

$T_{M A X}=4 \cdot F_{M A X}$

When the limit is speed is reached the acceleration is equal to zero and the maximum speed equation can be obtained as:

$$
v_{V}=\sqrt{\frac{2\left(T_{M A X}-m \cdot g\right)}{\rho_{A I R} \cdot C_{D} \cdot A}}
$$

Where $\mathrm{T}_{\mathrm{MAX}}$ : the maximum thrust force, $\mathrm{m}$ : the quadcopter mass, $\mathrm{g}$ : the earth gravity acceleration, A: the quadcopter area

\subsection{Maximum Horizontal Speed}

It is assumed that the quadcopter is moving only in the horizontal direction, keeping its altitude constant. It flies with an inclination angle, which can be obtained by compensating gravitational force with maximum thrust of propellers. After reaching the desired speed the acceleration is set to zero. At this instance the horizontal speed is represented as:

$v_{H}=\sqrt{\frac{2 \cdot m \cdot g \cdot \tan \alpha}{\rho_{A I R} \cdot C_{D} \cdot A \cdot \sin \alpha}}$

In order to control the quadcopter, as shown in Figure-4 we have used a PID control mechanism, with a component proportional to the error between our desired rate of change and the observed rate of change. Also a component proportional to the derivative of the rate of change. We can formulate this by enforcing the constraint that our inputs must keep the quadcopter aloft:

$\mathrm{T}=\mathrm{mg}$

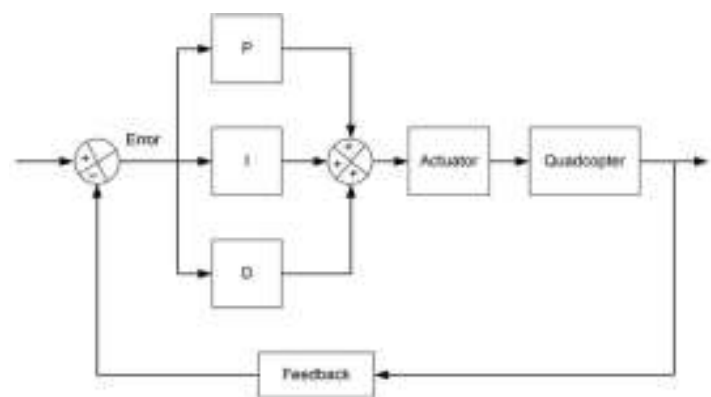

Figure-4: PID Mechanism for quadcopter

Our gyroscope is precise enough that we have integrated the values obtained from the quadcopter which is again used to calculate the thrust necessary to keep the quadcopter hover, by projecting the thrust $\mathrm{mg}$ onto the inertial $\mathrm{z}$ axis. Specifically for inclined surfaces, the quadcopter can fly easily. Therefore with a 
precise measurement using angle of friction, we have enforced the requirement that the thrust is equal to

$$
T=\frac{m g}{\cos \theta \cos \phi}
$$

Where $\theta$ and $\phi$ represents pitch angle and roll angle respectively.

\subsection{Maximum Flight Time}

The flight time is defined as the time while the battery is able to provide enough energy to the system. It depends on the battery capacity and on the current drawn by the motors and other electronic components. The current consumed by the motors depend on the required throttle that they have. Therefore, the flight time is variable and depends on the pilot requirements. However, it is possible to calculate the longest flight time that a battery can provide. The minimum current consumption is obtained in the situation that the quadcopter is hovering, as its motors only have to compensate for the system weight. For this situation the curren drawn by each motor can be calculated as

$T_{\text {Flig } \square t}=\frac{\text { Capacity }}{4 . I_{\text {MOTOR }}+I_{\text {OTHERS }}}$

Where "Capacity" is the capacity expressed in Amps per hour and " $\mathrm{T}_{\text {Flight }}$ " the flight time expressed in hours The component that consumes more current apart from the motors is the controller. Given the big scale difference, this current is assumed to be zero, resulting the simplified flight time in:

$T_{\text {Flig } \square t} \simeq \frac{\text { Capacity }}{4 . I_{\text {MOTOR }}}$

\section{Experimental Results and Performance Analysis}

To achieve mobility the quadcopter has been designed and tested in open air to overcome the drawback of inconsistency within a shorter range. For testing in real time the gamepad is connected to computer and the recorded value of the gamepad is transferred to the computer where the application is installed developed by Qt. This application is analyzing the value and sending to the smart phone. Another android application is also being installed in the smart phone which receives the value and sends it to the arduino micro controller which is again responsible for starting the rotors of the quadcopter. Then the Smartphone which is connected to the arduino is placed on the quadcopter and various sensors of the smart phone analyze the roll, yaw and pitch value of the quadcopter and send this value to the computer application where the application displays the value remotely. Also the camera of the smart phone is used to capture images, videos and forwards to the computer application to make the user aware about the remote location incidents. Table-1 shows the configuration component details of the mobile sink framework.

During hovering the quadcopter checks the obstacles due to ultrasonic sensors and changes its speed and angle. Also by getting the instruction from remote location the image can be captured by the mobile phone attached to the quadcopter and forwards to the destination machine through Wi-Fi or GSM module using internet. For the testing of data acquisition in the proposed framework the Smartphone uses its Wi-Fi for sending of data. So the quadcopter with necessary weight can be controlled through internet as both are connected to it. The request-response model is implemented manually from remote location through image capturing procedure. Also the location of quadcopter can be retrieved automatically or manually using GPS module which varies for different clock times by which it can be tracked during intra-city communication. Figure- 7 shows the change in longitude and latitude of quadcopter w.r.to time.

Table-1: Components of the designed mobile sink model

\begin{tabular}{|l|l|}
\hline Components & Description \\
\hline Microcontroller & ATmega2560 \\
\hline Operating Voltage & $5 \mathrm{~V}$ \\
\hline Input Voltage (recommended) & $7 \mathrm{~V}-12 \mathrm{~V}$ \\
\hline Input Voltage (limits) & $6 \mathrm{~V}-20 \mathrm{~V}$ \\
\hline Digital I/O Pins & $\begin{array}{l}54 \quad \text { of which 15 provide } \\
\text { PWM output) }\end{array}$ \\
\hline Analog Input Pins & 16 \\
\hline DC Current per I/O Pin & $40 \mathrm{~mA}$ \\
\hline DC Current for 3.3V Pin & $50 \mathrm{~mA}$ \\
\hline Flash Memory & $256 \mathrm{~KB}$ of which $8 \mathrm{~KB}$ used \\
& by boot loader \\
\hline SRAM & $8 \mathrm{~KB}$ \\
\hline EEPROM & $4 \mathrm{~KB}$ \\
\hline Clock Speed & $16 \mathrm{MHz}$ \\
\hline USB Host Chip & MAX3421E \\
\hline Length & $101.52 \mathrm{~mm}$ \\
\hline Width & $53.3 \mathrm{~mm}$ \\
\hline Weight & $36 \mathrm{~g}$ \\
\hline Operating system & Android \\
\hline Framework & Qt \\
\hline &
\end{tabular}

For testing of its effectiveness the framework has been used in real time through flight mode and data is captured through image and video as shown in figure-5. Similarly the image is be captured by the quadcoptor and forwarded through the developed application to the required machine or device of within the range. Here the black line of each graph represents the thrust which has to be always constant for keeping the quadcopter balanced in the medium during movement in a single direction. Whereas the blue line is the different values of corresponding angle to make adjustment while the quadcopter is in movement and captures the real time image from camera. The graph has been plotted through Qt by analyzing various angles with constant thrust versus time which gives different result in each second and displayed through figure-6.

Collected angles as data per sequence of seconds related to yaw, pitch and roll has been shown in Table-2 which is collected and tracked automatically through the GPS module. Data processing and forwarding is also managed by quadcopter from different angles to reach at destination and for communication with remote machine internet is used as the source.

For tracking the object from remote location GPS has been attached to the quadcopter and within time interval of one second the resultant latitude and longitude is being forwarded to the remote machine. Through this the quadcopter is being tracked from source to destination. As GPS provides the entire data with a larger string format, for our framework only the latitude and longitude values up to two digits are parsed for tracking and shown through figure-7. The values are automatically being stored in the database present in remote machine along with the time.

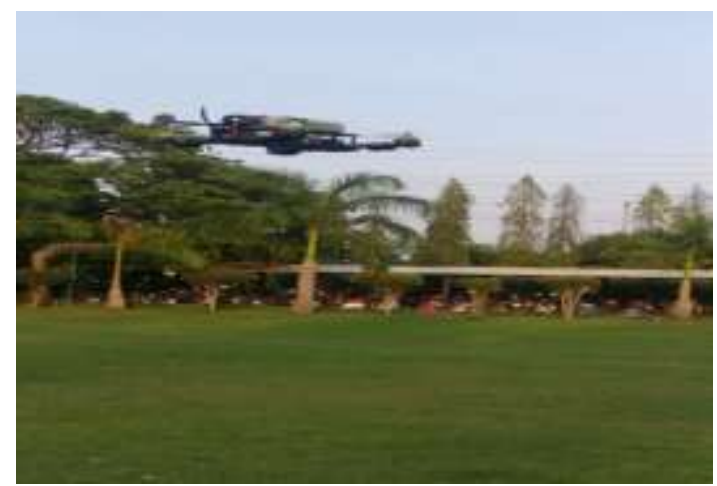

Figure5: Position of quadcoptor at different angle and altitude during travel 

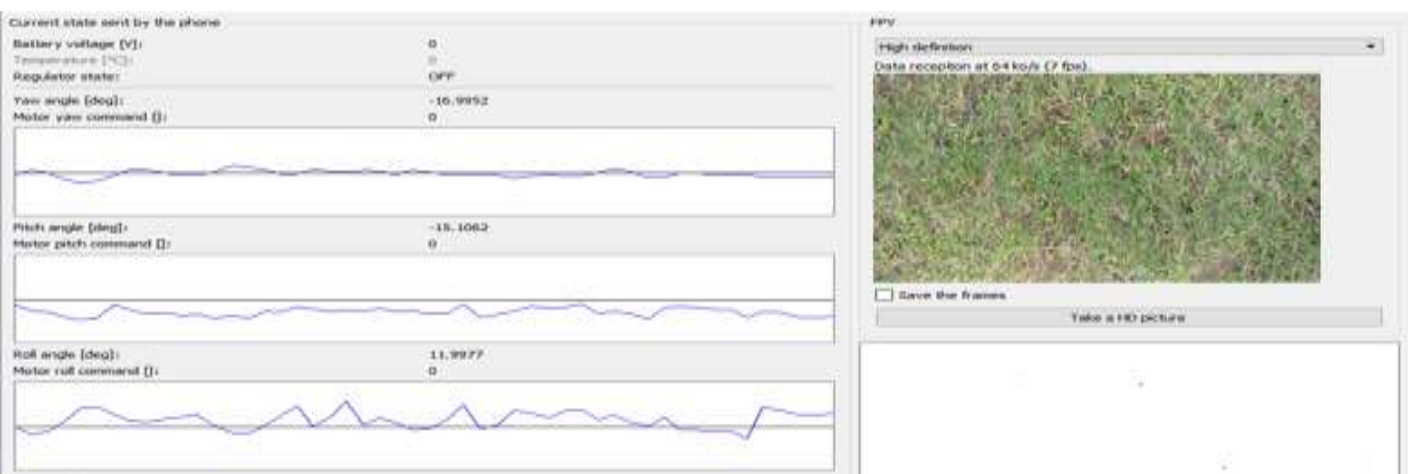

Figure 6: Capturing and processing of image through quad copter during hovering

Table -2: Collected angles in different sequences of seconds while moving

\begin{tabular}{|c|c|c|c|c|c|c|c|c|}
\hline TIME & $1: 57: 27$ & $1: 58: 14$ & $1: 58: 56$ & $1: 59: 06$ & $1: 59: 27$ & $1: 59: 34$ & $1: 59: 47$ & -10.73 \\
\hline YAW & -1.14 & -0.4 & -1.7 & -2.12 & 7.93 & -14.52 & -28.10 \\
\hline PITCH & -19.43 & -9.54 & -18.12 & -4.39 & -0.63 & -1.89 & -27.46 & -25.64 \\
\hline ROLL & -17.48 & -16.52 & -17.34 & -22.67 & -20.88 & -2.76 & -6.42 & -26.52 \\
\hline
\end{tabular}

\section{Conclusion}

The traditional way of using quadcopter is to control through wireless remote controller but to make the designed framework cost effective a gamepad has been used to control the quadcopter. Our main objective is to make use of remote device as transmitter and an android device as a receiver which is again effective compared with a transmitter receiver kit. So through our framework we have combined a smart phone with external hardware for collection of the real time data and processes for further remedies where human being cannot reach. The result shows the collection of image from height with different altitude in real time from remote location to provide real time assistance from anywhere. Also the computation task for data aggregation is done by the proposed framework effectively through wireless connectivity and low cost hardware along with communication through internet. But by using multiple quadcopters as mobile nodes can make the network more effective where data aggregation can be done locally for which lifetime of network can be longer. Like image the video of route can also be transferred to the remote machine using GSM module with static address. The real time event detection and processing can be achieved from remote location effectively through the framework and can be modified with multiple mobile sink.

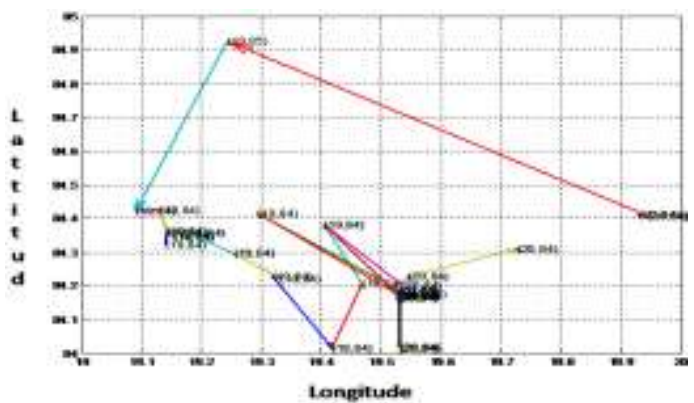

Figure-7: Real-Time latitude and longitude using GPS during movement

\section{Future Research Scope}

One of the major challenges for a mobile sink is to real-time delivery of the carrying item irrespective of environmental obstructions such as cyclone, whirlwind, raining, fog, night time etc. In addition to the air-thrust and other obstacles the high flying birds and their unpredictable appearance from any direction is another challenge in drone design. Statistically a mobile sink utilizes maximum battery power for its fly rather than carrying any item. With the existing battery technologies the movement of the sink might be restricted to certain distance only instead of reaching to the final destination. Another scope of this model is to integrate geographical positioning system and auto-navigation to ensure security breach and cross-border issue at major boundaries. A potential direction of research could be online flight planning and management, a reliable internet connection without http, collaborative vehicle-to-vehicle sense-and-avoid systems to avoid hazards. Further scope of improvement in the model is to handle seamless connection establishment of mobile sink with the required targets during intermittent of GPS connecting channels. In an enterprise network more number of interconnected mobile sinks may be provisioned for sink-to-sink communication and for auto-avoidance from cross-through obstructions. Although all of the challenges mentioned above can be provided separately, but the real-challenge to make the model robust lies in the performance of the model, in the real-field with satisfying desired requirement with the presence of unwanted obstructions by enhancing battery lifetime.

\section{References}

[1] Achtelik, Markus, et al. "Visual tracking and control of a quadcopter using a stereo camera system and inertial sensors." Mechatronics and automation, 2009. icma 2009. international conference on. IEEE, 2009.

[2] Bachrach, Abraham, et al. "RANGE-Robust autonomous navigation in GPS-denied environments." Journal of Field Robotics 28.5 (2011): 644-666.

[3] Moranduzzo, Thomas, and Farid Melgani. "Automatic car counting method for unmanned aerial vehicle images." IEEE Transactions on Geoscience and Remote Sensing 52.3 (2014): 1635-1647.

[4] Carrillo, LR Garcia, Alejandro Dzul, and Rogelio Lozano. "Hovering quad-rotor control: A comparison of nonlinear controllers using visual feedback." IEEE Transactions on Aerospace and Electronic Systems 48.4 (2012): 3159-3170.

[5] Zhang, Tianguang, et al. "Multi-sensory motion estimation and control of a mini-quadrotor in an air-ground multi-robot system." Robotics and Biomimetics (ROBIO), 2009 IEEE International Conference on. IEEE, 2009.

[6] Jeong, S. H., Jung, S., \& Tomizuka, M. (2012, July). Attitude control of a quad-rotor system using an acceleration-based disturbance observer: An empirical approach. In Advanced Intelligent Mechatronics (AIM), 2012 IEEE/ASME International Conference : $916-921$

[7] Kougianos, E., Mohanty, S. P., Coelho, G., Albalawi, U., \& Sundaravadivel, P. (2016). Design of a high-performance system for secure image communication in the Internet of Things. IEEE Access, 4, 1222-1242. 
[8] Chen N, Chen Y, You Y, Ling H, Liang P, Zimmermann R. Dynamic urban surveillance video stream processing using fog computing. InMultimedia Big Data (BigMM), 2016 IEEE Second International Conference on 2016 Apr 20 (pp. 105-112). IEEE.

[9] Kim, Byung Hyung, Minho Kim, and Sungho Jo. "Quadcopter flight control using a low-cost hybrid interface with EEG-based classification and eye tracking." Computers in biology and medicine 51 (2014): 82-92.

[10] Zhao, Weihua, and Tiauw Hiong Go. "Quadcopter formation flight control combining MPC and robust feedback linearization." Journal of the Franklin Institute 351.3 (2014): 1335-1355.

[11] Kumar, Prem, et al. "Measurement of Power Radiation in Base Transceiver Station Using Quad Phone and Quadcopter." Journal of Green Engineering 5.2 (2015): 107-128.

[12] Rajpoot, Anurag Singh, Namrata Gadani, and Sagar Kalathia "Development of Arduino Based Quadcopter." International Advanced Research Journal in Science, Engineering and Technology 3.6 (2016).

[13] Leong, Bernard Tat Meng, Sew Ming Low, and Melanie Po-Leen Ooi. "Low-cost microcontroller-based hover control design of a quadcopter." Procedia Engineering 41 (2012): 458-464.

[14] Vechian, Mongkhun Qetkeaw. Wireless control quadcopter with stereo camera and self-balancing system. Diss. Universiti Tun Hussein Onn Malaysia, 2012.

[15] Ji, Ankyd, and Kamran Turkoglu. "Development of a low-cost experimental quadcopter testbed using an arduino controller for video surveillance." AIAA Infotech@ Aerospace (2015).

[16] Papa, Umberto, and Giuseppe Del Core. "Design and Assembling of a low-cost Mini UAV Quadcopter System." Department of Science and Technology, University of Naples" Parthenope(2014).

[17] Huston, Dryver R., Dylan Burns, and Mandar M. Dewoolkar. "Integration of Automated and Robotic Systems with BIM for Comprehensive Structural Assessment." Structures Congress 2014. 2014.

[18] Nayak, S. P., Dhal, K., Rai, S. C., \& Pradhan, S. K. (2015, September). TIME: Supporting topology independent mobility with energy efficient routing in WSNs. In Next Generation Computing Technologies (NGCT), 2015 1st International Conference on (pp. 350-355). IEEE.

[19] Raval, G., \& Bhavsar, M. (2015). Improving energy estimation based clustering with energy threshold for wireless sensor networks. International Journal of Computer Applications, 113(19).

[20] Hong, Yu-Jin, et al. "Mobile health monitoring system based on activity recognition using accelerometer." Simulation Modelling Practice and Theory 18.4 (2010): 446-455.

[21] Nayak, S. P., Rai, S. C., \& Pradhan, S. K. (2015, December) MERA: A Multi-clustered Energy Efficient Routing Algorithm in WSN. In Information Technology (ICIT), 2015 International Conference on (pp. 37-42). IEEE.

[22] Doukas, Charalampos, and Ilias Maglogiannis. "Bringing IoT and cloud computing towards pervasive healthcare." In Innovative Mobile and Internet Services in Ubiquitous Computing (IMIS), 2012 Sixth International Conference on, pp. 922-926. IEEE, 2012.

[23] Istepanian, R. S., Hu, S., Philip, N. Y., \& Sungoor, A. (2011, August). The potential of Internet of $\mathrm{m}$-health Things " $\mathrm{m}$-IoT" for non-invasive glucose level sensing. In Engineering in Medicine and Biology Society, EMBC, 2011 Annual International Conference of the IEEE (pp. 5264-5266). IEEE.

[24] Atzori, Luigi, Antonio Iera, and Giacomo Morabito. "The internet of things: A survey." Computer networks 54, no. 15 (2010): 2787-2805.

[25] Hassanalieragh, Moeen, Alex Page, Tolga Soyata, Gaurav Sharma, Mehmet Aktas, Gonzalo Mateos, Burak Kantarci, and Silvana Andreescu. "Health monitoring and management using Internet-ofThings (IoT) sensing with cloud-based processing: Opportunities and challenges." In Services Computing (SCC), 2015 IEEE International Conference on, pp. 285-292. IEEE, 2015. 